\title{
Search for Standard Model Higgs Bosons Produced in Association with $W$ Bosons
}

T. Aaltonen, ${ }^{23}$ J. Adelman, ${ }^{13}$ T. Akimoto, ${ }^{54}$ M. G. Albrow, ${ }^{17}$ B. Álvarez González, ${ }^{11}$ S. Amerio, ${ }^{42}$ D. Amidei, ${ }^{34}$ A. Anastassov ${ }^{51}$ A. Annovi, ${ }^{19}$ J. Antos, ${ }^{14}$ M. Aoki,${ }^{24}$ G. Apollinari, ${ }^{17}$ A. Apresyan, ${ }^{47}$ T. Arisawa, ${ }^{56}$ A. Artikov, ${ }^{15}$ W. Ashmanskas, ${ }^{17}$ A. Attal, ${ }^{3}$ A. Aurisano, ${ }^{52}$ F. Azfar, ${ }^{41}$ P. Azzi-Bacchetta, ${ }^{42}$ P. Azzurri, ${ }^{45}$ N. Bacchetta, ${ }^{42}$ W. Badgett, ${ }^{17}$ A. Barbaro-Galtieri, ${ }^{28}$ V.E. Barnes, ${ }^{47}$ B. A. Barnett, ${ }^{25}$ S. Baroiant,${ }^{7}$ V. Bartsch, ${ }^{30}$ G. Bauer, ${ }^{32}$ P.-H. Beauchemin, ${ }^{33}$ F. Bedeschi, ${ }^{45}$ P. Bednar, ${ }^{14}$ S. Behari,${ }^{25}$ G. Bellettini, ${ }^{45}$ J. Bellinger, ${ }^{58}$ A. Belloni, ${ }^{22}$ D. Benjamin, ${ }^{16}$ A. Beretvas, ${ }^{17}$ J. Beringer ${ }^{28}$ T. Berry, ${ }^{29}$ A. Bhatti, ${ }^{49}$ M. Binkley, ${ }^{17}$ D. Bisello, ${ }^{42}$ I. Bizjak, ${ }^{30}$ R. E. Blair, ${ }^{2}$ C. Blocker, ${ }^{6}$ B. Blumenfeld ${ }^{25}$ A. Bocci, ${ }^{16}$ A. Bodek, ${ }^{48}$ V. Boisvert, ${ }^{48}$ G. Bolla, ${ }^{47}$ A. Bolshov, ${ }^{32}$ D. Bortoletto, ${ }^{47}$ J. Boudreau, ${ }^{46}$ A. Boveia, ${ }^{10}$ B. Brau,${ }^{10}$ A. Bridgeman, ${ }^{24}$ L. Brigliadori, ${ }^{5}$ C. Bromberg, ${ }^{35}$ E. Brubaker, ${ }^{13}$ J. Budagov, ${ }^{15}$ H. S. Budd, ${ }^{48}$ S. Budd,${ }^{24}$ K. Burkett, ${ }^{17}$ G. Busetto, ${ }^{42}$ P. Bussey, ${ }^{21}$ A. Buzatu, ${ }^{33}$ K. L. Byrum, ${ }^{2}$ S. Cabrera, ${ }^{16, s}$ M. Campanelli, ${ }^{35}$ M. Campbell, ${ }^{34}$ F. Canelli, ${ }^{17}$ A. Canepa, ${ }^{44}$ D. Carlsmith, ${ }^{58}$ R. Carosi, ${ }^{45}$ S. Carrillo, ${ }^{18, m}$ S. Carron, ${ }^{33}$ B. Casal,${ }^{11}$ M. Casarsa, ${ }^{17}$ A. Castro,${ }^{5}$ P. Catastini,${ }^{45}$ D. Cauz, ${ }^{53}$ M. Cavalli-Sforza, ${ }^{3}$ A. Cerri, ${ }^{28}$ L. Cerrito, ${ }^{30, q}$ S. H. Chang, ${ }^{27}$ Y. C. Chen, ${ }^{1}$ M. Chertok, ${ }^{7}$ G. Chiarelli, ${ }^{45}$ G. Chlachidze, ${ }^{17}$ F. Chlebana, ${ }^{17}$ K. Cho, ${ }^{27}$ D. Chokheli, ${ }^{15}$ J. P. Chou, ${ }^{22}$ G. Choudalakis, ${ }^{32}$ S. H. Chuang, ${ }^{51}$ K. Chung, ${ }^{12}$ W. H. Chung, ${ }^{58}$ Y. S. Chung, ${ }^{48}$ C. I. Ciobanu,${ }^{24}$ M. A. Ciocci, ${ }^{45}$ A. Clark, ${ }^{20}$ D. Clark, ${ }^{6}$ G. Compostella, ${ }^{42}$ M. E. Convery, ${ }^{17}$ J. Conway, ${ }^{7}$ B. Cooper, ${ }^{30}$ K. Copic,${ }^{34}$ M. Cordelli, ${ }^{19}$ G. Cortiana, ${ }^{42}$ F. Crescioli, ${ }^{45}$ C. Cuenca Almenar, ${ }^{7, \mathrm{~s}}$ J. Cuevas, ${ }^{11, p}$ R. Culbertson, ${ }^{17}$ J. C. Cully, ${ }^{34}$ D. Dagenhart, ${ }^{17}$ M. Datta, ${ }^{17}$ T. Davies, ${ }^{21}$ P. de Barbaro, ${ }^{48}$ S. De Cecco, ${ }^{50}$ A. Deisher, ${ }^{28}$ G. De Lentdecker, ${ }^{48, e}$ G. De Lorenzo,${ }^{3}$ M. Dell'Orso, ${ }^{45}$ L. Demortier, ${ }^{49}$ J. Deng, ${ }^{16}$ M. Deninno, ${ }^{5}$ D. De Pedis, ${ }^{50}$

P. F. Derwent, ${ }^{17}$ G. P. Di Giovanni,${ }^{43}$ C. Dionisi,${ }^{50}$ B. Di Ruzza,${ }^{53}$ J. R. Dittmann, ${ }^{4}$ M. D'Onofrio, ${ }^{3}$ S. Donati, ${ }^{45}$ P. Dong, ${ }^{8}$ J. Donini, ${ }^{42}$ T. Dorigo,${ }^{42}$ S. Dube, ${ }^{51}$ J. Efron, ${ }^{38}$ R. Erbacher, ${ }^{7}$ D. Errede,${ }^{24}$ S. Errede, ${ }^{24}$ R. Eusebi, ${ }^{17}$ H. C. Fang, ${ }^{28}$

S. Farrington, ${ }^{29}$ W. T. Fedorko, ${ }^{13}$ R. G. Feild,${ }^{59}$ M. Feindt, ${ }^{26}$ J. P. Fernandez,${ }^{31}$ C. Ferrazza, ${ }^{45}$ R. Field, ${ }^{18}$ G. Flanagan, ${ }^{47}$ R. Forrest, ${ }^{7}$ S. Forrester, ${ }^{7}$ M. Franklin, ${ }^{22}$ J. C. Freeman, ${ }^{28}$ I. Furic, ${ }^{18}$ M. Gallinaro, ${ }^{49}$ J. Galyardt, ${ }^{12}$ F. Garberson, ${ }^{10}$ J.E. Garcia, ${ }^{45}$ A. F. Garfinkel, ${ }^{47}$ H. Gerberich,${ }^{24}$ D. Gerdes,${ }^{34}$ S. Giagu, ${ }^{50}$ V. Giakoumopolou, ${ }^{45, b}$ P. Giannetti, ${ }^{45}$ K. Gibson, ${ }^{46}$ J. L. Gimmell, ${ }^{48}$ C. M. Ginsburg,,${ }^{17}$ N. Giokaris, ${ }^{15, b}$ M. Giordani, ${ }^{53}$ P. Giromini, ${ }^{19}$ M. Giunta, ${ }^{45}$ V. Glagolev, ${ }^{15}$ D. Glenzinski, ${ }^{17}$ M. Gold, ${ }^{36}$ N. Goldschmidt, ${ }^{18}$ A. Golossanov, ${ }^{17}$ G. Gomez, ${ }^{11}$ G. Gomez-Ceballos, ${ }^{32}$ M. Goncharov, ${ }^{52}$ O. González, ${ }^{31}$ I. Gorelov, ${ }^{36}$ A. T. Goshaw ${ }^{16}$ K. Goulianos, ${ }^{49}$ A. Gresele, ${ }^{42}$ S. Grinstein, ${ }^{22}$ C. Grosso-Pilcher, ${ }^{13}$

R. C. Group, ${ }^{17}$ U. Grundler, ${ }^{24}$ J. Guimaraes da Costa, ${ }^{22}$ Z. Gunay-Unalan, ${ }^{35}$ C. Haber, ${ }^{28}$ K. Hahn, ${ }^{32}$ S. R. Hahn, ${ }^{17}$ E. Halkiadakis, ${ }^{51}$ A. Hamilton, ${ }^{20}$ B.-Y. Han, ${ }^{48}$ J. Y. Han, ${ }^{48}$ R. Handler, ${ }^{58}$ F. Happacher, ${ }^{19}$ K. Hara,${ }^{54}$ D. Hare, ${ }^{51}$ M. Hare,${ }^{55}$ S. Harper ${ }^{41}$ R. F. Harr, ${ }^{57}$ R. M. Harris,${ }^{17}$ M. Hartz,${ }^{46}$ K. Hatakeyama, ${ }^{49}$ J. Hauser, ${ }^{8}$ C. Hays, ${ }^{41}$ M. Heck,${ }^{26}$ A. Heijboer,${ }^{44}$ B. Heinemann, ${ }^{28}$ J. Heinrich, ${ }^{44}$ C. Henderson, ${ }^{32}$ M. Herndon, ${ }^{58}$ J. Heuser, ${ }^{26}$ S. Hewamanage, ${ }^{4}$ D. Hidas,${ }^{16}$ C. S. Hill, ${ }^{10, d}$ D. Hirschbuehl, ${ }^{26}$ A. Hocker, ${ }^{17}$ S. Hou, ${ }^{1}$ M. Houlden, ${ }^{29}$ S.-C. Hsu, ${ }^{9}$ B. T. Huffman, ${ }^{41}$ R. E. Hughes,${ }^{38}$ U. Husemann,${ }^{59}$ J. Huston, ${ }^{35}$ J. Incandela,${ }^{10}$ G. Introzzi ${ }^{45}$ M. Iori, ${ }^{50}$ A. Ivanov, ${ }^{7}$ B. Iyutin, ${ }^{32}$ E. James, ${ }^{17}$ B. Jayatilaka, ${ }^{16}$ D. Jeans,${ }^{50}$ E. J. Jeon, ${ }^{27}$ S. Jindariani, ${ }^{18}$ W. Johnson, ${ }^{7}$ M. Jones, ${ }^{47}$ K. K. Joo, ${ }^{27}$ S. Y. Jun, ${ }^{12}$ J. E. Jung, ${ }^{27}$ T. R. Junk, ${ }^{24}$ T. Kamon, ${ }^{52}$ D. Kar, ${ }^{18}$ P.E. Karchin,${ }^{57}$ Y. Kato,${ }^{40}$ R. Kephart, ${ }^{17}$ U. Kerzel, ${ }^{26}$ V. Khotilovich,${ }^{52}$ B. Kilminster, ${ }^{38}$ D. H. Kim, ${ }^{27}$ H. S. Kim ${ }^{27}$ J.E. Kim, ${ }^{27}$ M. J. Kim,${ }^{17}$ S. B. Kim,${ }^{27}$ S. H. Kim,${ }^{54}$ Y. K. Kim,${ }^{13}$ N. Kimura,${ }^{54}$ L. Kirsch, ${ }^{6}$ S. Klimenko, ${ }^{18}$ M. Klute,${ }^{32}$ B. Knuteson, ${ }^{32}$ B. R. Ko, ${ }^{16}$ S. A. Koay, ${ }^{10}$ K. Kondo, ${ }^{56}$ D. J. Kong, ${ }^{27}$ J. Konigsberg, ${ }^{18}$ A. Korytov, ${ }^{18}$

A. V. Kotwal, ${ }^{16}$ J. Kraus,${ }^{24}$ M. Kreps, ${ }^{26}$ J. Kroll,${ }^{44}$ N. Krumnack,${ }^{4}$ M. Kruse,${ }^{16}$ V. Krutelyov, ${ }^{10}$ T. Kubo ${ }^{54}$ S. E. Kuhlmann, ${ }^{2}$

T. Kuhr ${ }^{26}$ N. P. Kulkarni, ${ }^{57}$ Y. Kusakabe, ${ }^{56}$ S. Kwang, ${ }^{13}$ A. T. Laasanen, ${ }^{47}$ S. Lai, ${ }^{33}$ S. Lami, ${ }^{45}$ S. Lammel,${ }^{17}$

M. Lancaster ${ }^{30}$ R. L. Lander, ${ }^{7}$ K. Lannon, ${ }^{38}$ A. Lath, ${ }^{51}$ G. Latino, ${ }^{45}$ I. Lazzizzera, ${ }^{42}$ T. LeCompte, ${ }^{2}$ J. Lee, ${ }^{48}$ J. Lee, ${ }^{27}$ Y. J. Lee, ${ }^{27}$ S. W. Lee, ${ }^{52, r}$ R. Lefèvre, ${ }^{20}$ N. Leonardo, ${ }^{32}$ S. Leone, ${ }^{45}$ S. Levy, ${ }^{13}$ J. D. Lewis, ${ }^{17}$ C. Lin, ${ }^{59}$ C. S. Lin, ${ }^{28}$ J. Linacre ${ }^{41}$ M. Lindgren, ${ }^{17}$ E. Lipeles, ${ }^{9}$ A. Lister, ${ }^{7}$ D. O. Litvintsev, ${ }^{17}$ T. Liu, ${ }^{17}$ N. S. Lockyer, ${ }^{44}$ A. Loginov, ${ }^{59}$ M. Loreti, ${ }^{42}$ L. Lovas, ${ }^{14}$ R.-S. Lu, ${ }^{1}$ D. Lucchesi, ${ }^{42}$ J. Lueck, ${ }^{26}$ C. Luci, ${ }^{50}$ P. Lujan, ${ }^{28}$ P. Lukens, ${ }^{17}$ G. Lungu, ${ }^{18}$ L. Lyons, ${ }^{41}$ J. Lys, ${ }^{28}$ R. Lysak, ${ }^{14}$ E. Lytken,${ }^{47}$ P. Mack, ${ }^{26}$ D. MacQueen, ${ }^{33}$ R. Madrak,${ }^{17}$ K. Maeshima,,${ }^{17}$ K. Makhoul, ${ }^{32}$ T. Maki, ${ }^{23}$ P. Maksimovic ${ }^{25}$ S. Malde,${ }^{41}$ S. Malik, ${ }^{30}$ G. Manca, ${ }^{29}$ A. Manousakis, ${ }^{15, b}$ F. Margaroli, ${ }^{47}$ C. Marino, ${ }^{26}$ C. P. Marino, ${ }^{24}$ A. Martin, ${ }^{59}$ M. Martin, ${ }^{25}$ V. Martin, ${ }^{21, \mathrm{k}}$ M. Martínez, ${ }^{3}$ R. Martínez-Ballarín, ${ }^{31}$ T. Maruyama,${ }^{54}$ P. Mastrandrea, ${ }^{50}$ T. Masubuchi,${ }^{54}$ M. E. Mattson, ${ }^{57}$ P. Mazzanti, ${ }^{5}$ K. S. McFarland,${ }^{48}$ P. McIntyre, ${ }^{52}$ R. McNulty, ${ }^{29, j}$ A. Mehta, ${ }^{29}$ P. Mehtala ${ }^{23}$ S. Menzemer, ${ }^{11,1}$ A. Menzione, ${ }^{45}$ P. Merkel,${ }^{47}$ C. Mesropian,${ }^{49}$ A. Messina,${ }^{35}$ T. Miao,${ }^{17}$ N. Miladinovic,${ }^{6}$ J. Miles,${ }^{32}$ R. Miller, ${ }^{35}$ C. Mills, ${ }^{22}$ M. Milnik, ${ }^{26}$ A. Mitra, ${ }^{1}$ G. Mitselmakher, ${ }^{18}$ H. Miyake, ${ }^{54}$ S. Moed, ${ }^{22}$ N. Moggi, ${ }^{5}$ C. S. Moon, ${ }^{27}$ R. Moore,${ }^{17}$ M. Morello ${ }^{45}$ P. Movilla Fernandez, ${ }^{28}$ J. Mülmenstädt ${ }^{28}$ A. Mukherjee,${ }^{17}$ Th. Muller, ${ }^{26}$ R. Mumford, ${ }^{25}$ P. Murat,${ }^{17}$ M. Mussini,${ }^{5}$ J. Nachtman, ${ }^{17}$ Y. Nagai, ${ }^{54}$ A. Nagano, ${ }^{54}$ J. Naganoma, ${ }^{56}$ K. Nakamura, ${ }^{54}$ 
I. Nakano, ${ }^{39}$ A. Napier, ${ }^{55}$ V. Necula, ${ }^{16}$ C. Neu, ${ }^{44}$ M. S. Neubauer, ${ }^{24}$ J. Nielsen, ${ }^{28, g}$ L. Nodulman, ${ }^{2}$ M. Norman, ${ }^{9}$ O. Norniella ${ }^{24}$ E. Nurse ${ }^{30}$ S. H. Oh, ${ }^{16}$ Y. D. Oh,${ }^{27}$ I. Oksuzian, ${ }^{18}$ T. Okusawa ${ }^{40}$ R. Oldeman, ${ }^{29}$ R. Orava, ${ }^{23}$ K. Osterberg,${ }^{23}$ S. Pagan Griso, ${ }^{42}$ C. Pagliarone,${ }^{45}$ E. Palencia, ${ }^{17}$ V. Papadimitriou, ${ }^{17}$ A. Papaikonomou ${ }^{26}$ A. A. Paramonov,${ }^{13}$ B. Parks,${ }^{38}$ S. Pashapour, ${ }^{33}$ J. Patrick, ${ }^{17}$ G. Pauletta, ${ }^{53}$ M. Paulini, ${ }^{12}$ C. Paus, ${ }^{32}$ D. E. Pellett, ${ }^{7}$ A. Penzo,${ }^{53}$ T. J. Phillips, ${ }^{16}$ G. Piacentino, ${ }^{45}$ J. Piedra, ${ }^{43}$ L. Pinera,${ }^{18}$ K. Pitts, ${ }^{24}$ C. Plager, ${ }^{8}$ L. Pondrom,${ }^{58}$ X. Portell, ${ }^{3}$ O. Poukhov, ${ }^{15}$ N. Pounder,${ }^{41}$ F. Prakoshyn, ${ }^{15}$ A. Pronko, ${ }^{17}$ J. Proudfoot, ${ }^{2}$ F. Ptohos, ${ }^{17, i}$ G. Punzi, ${ }^{45}$ J. Pursley, ${ }^{58}$ J. Rademacker, ${ }^{41, d}$ A. Rahaman, ${ }^{46}$ V. Ramakrishnan, ${ }^{58}$ N. Ranjan, ${ }^{47}$ I. Redondo, ${ }^{31}$ B. Reisert, ${ }^{17}$ V. Rekovic, ${ }^{36}$ P. Renton, ${ }^{41}$ M. Rescigno, ${ }^{50}$ S. Richter, ${ }^{26}$ F. Rimondi, ${ }^{5}$ L. Ristori ${ }^{45}$ A. Robson, ${ }^{21}$ T. Rodrigo,${ }^{11}$ E. Rogers,${ }^{24}$ S. Rolli, ${ }^{55}$ R. Roser, ${ }^{17}$ M. Rossi, ${ }^{53}$ R. Rossin, ${ }^{10}$ P. Roy,${ }^{33}$ A. Ruiz, ${ }^{11}$ J. Russ, ${ }^{12}$ V. Rusu, ${ }^{17}$ H. Saarikko, ${ }^{23}$ A. Safonov, ${ }^{52}$ W. K. Sakumoto, ${ }^{48}$ G. Salamanna,${ }^{50}$ O. Saltó, ${ }^{3}$ L. Santi, ${ }^{53}$ S. Sarkar, ${ }^{50}$ L. Sartori, ${ }^{45}$ K. Sato, ${ }^{17}$ A. Savoy-Navarro, ${ }^{43}$ T. Scheidle, ${ }^{26}$ P. Schlabach, ${ }^{17}$ E. E. Schmidt, ${ }^{17}$ M. A. Schmidt, ${ }^{13}$ M.P. Schmidt, ${ }^{59}$ M. Schmitt, ${ }^{37}$ T. Schwarz, ${ }^{7}$ L. Scodellaro, ${ }^{11}$ A. L. Scott, ${ }^{10}$ A. Scribano, ${ }^{45}$ F. Scuri, ${ }^{45}$ A. Sedov, ${ }^{47}$ S. Seidel,${ }^{36}$ Y. Seiya ${ }^{40}$ A. Semenov,${ }^{15}$ L. Sexton-Kennedy, ${ }^{17}$ A. Sfyria, ${ }^{20}$ S. Z. Shalhout, ${ }^{57}$ M. D. Shapiro, ${ }^{28}$ T. Shears ${ }^{29}$ P. F. Shepard, ${ }^{46}$ D. Sherman, ${ }^{22}$ M. Shimojima, ${ }^{54,0}$ M. Shochet, ${ }^{13}$ Y. Shon, ${ }^{58}$ I. Shreyber, ${ }^{20}$ A. Sidoti, ${ }^{45}$ J. Siegrist, ${ }^{28}$ P. Sinervo, ${ }^{33}$ A. Sisakyan, ${ }^{15}$ A. J. Slaughter, ${ }^{17}$ J. Slaunwhite, ${ }^{38}$ K. Sliwa, ${ }^{55}$ J. R. Smith, ${ }^{7}$ F. D. Snider, ${ }^{17}$ R. Snihur, ${ }^{33}$ M. Soderberg, ${ }^{34}$ A. Soha, ${ }^{7}$ S. Somalwar, ${ }^{51}$ V. Sorin, ${ }^{35}$ J. Spalding, ${ }^{17}$ F. Spinella, ${ }^{45}$ T. Spreitzer, ${ }^{33}$ P. Squillacioti, ${ }^{45}$ M. Stanitzki, ${ }^{59}$ R. St. Denis, ${ }^{21}$ B. Stelzer, ${ }^{8}$ O. Stelzer-Chilton, ${ }^{41}$ D. Stentz, ${ }^{37}$ J. Strologas, ${ }^{36}$ D. Stuart, ${ }^{10}$ J. S. Suh, ${ }^{27}$ A. Sukhanov, ${ }^{18}$ H. Sun, ${ }^{55}$ I. Suslov ${ }^{15}$ T. Suzuki, ${ }^{54}$ A. Taffard,${ }^{24, f}$ R. Takashima, ${ }^{39}$ Y. Takeuchi, ${ }^{54}$ R. Tanaka, ${ }^{39}$ M. Tecchio, ${ }^{34}$ P. K. Teng, ${ }^{1}$ K. Terashi,${ }^{49}$ J. Thom, ${ }^{17, h}$ A. S. Thompson, ${ }^{21}$ G. A. Thompson, ${ }^{24}$ E. Thomson, ${ }^{44}$ P. Tipton, ${ }^{59}$ V. Tiwari, ${ }^{12}$ S. Tkaczyk, ${ }^{17}$ D. Toback,${ }^{52}$ S. Tokar,${ }^{14}$ K. Tollefson,${ }^{35}$ T. Tomura, ${ }^{54}$ D. Tonelli, ${ }^{17}$ S. Torre, ${ }^{19}$ D. Torretta, ${ }^{17}$ S. Tourneur, ${ }^{43}$ W. Trischuk ${ }^{33}$ Y. Tu, ${ }^{44}$ N. Turini,${ }^{45}$ F. Ukegawa, ${ }^{54}$ S. Uozumi,${ }^{54}$ S. Vallecorsa ${ }^{20}$ N. van Remortel, ${ }^{23}$ A. Varganov, ${ }^{34}$ E. Vataga,${ }^{36}$ F. Vázquez, ${ }^{18, \mathrm{~m}} \mathrm{G}$. Velev,${ }^{17} \mathrm{C}$. Vellidis, ${ }^{45, \mathrm{~b}}$ V. Veszpremi, ${ }^{47} \mathrm{M}$. Vidal,${ }^{31} \mathrm{R}$. Vidal, ${ }^{17} \mathrm{I}$. Vila, ${ }^{11}$ R. Vilar, ${ }^{11}$ T. Vine, ${ }^{30}$ M. Vogel,${ }^{36}$ I. Volobouev, ${ }^{28, r}$ G. Volpi, ${ }^{45}$ F. Würthwein, ${ }^{9}$ P. Wagner, ${ }^{44}$ R. G. Wagner, ${ }^{2}$ R. L. Wagner, ${ }^{17}$ J. Wagner-Kuhr, ${ }^{26}$ W. Wagner, ${ }^{26}$ T. Wakisaka ${ }^{40}$ R. Wallny, ${ }^{8}$ S. M. Wang, ${ }^{1}$ A. Warburton, ${ }^{33}$ D. Waters, ${ }^{30}$ M. Weinberger, ${ }^{52}$ W. C. Wester III, ${ }^{17}$ B. Whitehouse, ${ }^{55}$ D. Whiteson, ${ }^{44, f}$ A. B. Wicklund, ${ }^{2}$ E. Wicklund, ${ }^{17}$ G. Williams, ${ }^{33}$ H. H. Williams, ${ }^{44}$ P. Wilson, ${ }^{17}$ B. L. Winer, ${ }^{38}$ P. Wittich, ${ }^{17, h}$ S. Wolbers,${ }^{17}$ C. Wolfe, ${ }^{13}$ T. Wright,${ }^{34}$ X. Wu ${ }^{20}$ S. M. Wynne,${ }^{29}$ A. Yagil,${ }^{9}$ K. Yamamoto, ${ }^{40}$ J. Yamaoka, ${ }^{51}$ T. Yamashita, ${ }^{39}$ C. Yang, ${ }^{59}$ U. K. Yang, ${ }^{13, n}$ Y. C. Yang, ${ }^{27}$ W. M. Yao, ${ }^{28}$ G. P. Yeh, ${ }^{17}$ J. Yoh, ${ }^{17}$ K. Yorita, ${ }^{13}$ T. Yoshida, ${ }^{40}$ G. B. Yu, ${ }^{48}$ I. Yu, ${ }^{27}$ S. S. Yu, ${ }^{17}$ J. C. Yun, ${ }^{17}$ L. Zanello, ${ }^{50}$ A. Zanetti, ${ }^{53}$ I. Zaw, ${ }^{22}$ X. Zhang, ${ }^{24}$ Y. Zheng, ${ }^{8, c}$ and S. Zucchelli ${ }^{5}$

\title{
$(\mathrm{CDF} \text { Collaboration })^{1}$
}

\author{
${ }^{1}$ Institute of Physics, Academia Sinica, Taipei, Taiwan 11529, Republic of China \\ ${ }^{2}$ Argonne National Laboratory, Argonne, Illinois 60439, USA \\ ${ }^{3}$ Institut de Fisica d'Altes Energies, Universitat Autonoma de Barcelona, E-08193, Bellaterra (Barcelona), Spain \\ ${ }^{4}$ Baylor University, Waco, Texas 76798, USA \\ ${ }^{5}$ Istituto Nazionale di Fisica Nucleare, University of Bologna, I-40127 Bologna, Italy \\ ${ }^{6}$ Brandeis University, Waltham, Massachusetts 02254, USA \\ ${ }^{7}$ University of California, Davis, Davis, California 95616, USA \\ ${ }^{8}$ University of California, Los Angeles, Los Angeles, California 90024, USA \\ ${ }^{9}$ University of California, San Diego, La Jolla, California 92093, USA \\ ${ }^{10}$ University of California, Santa Barbara, Santa Barbara, California 93106, USA \\ ${ }^{11}$ Instituto de Fisica de Cantabria, CSIC-University of Cantabria, 39005 Santander, Spain \\ ${ }^{12}$ Carnegie Mellon University, Pittsburgh, Pennsylvania 15213, USA \\ ${ }^{13}$ Enrico Fermi Institute, University of Chicago, Chicago, Illinois 60637, USA \\ ${ }^{14}$ Comenius University, 84248 Bratislava, Slovakia; Institute of Experimental Physics, 04001 Kosice, Slovakia \\ ${ }^{15}$ Joint Institute for Nuclear Research, RU-141980 Dubna, Russia \\ ${ }^{16}$ Duke University, Durham, North Carolina 27708, USA \\ ${ }^{17}$ Fermi National Accelerator Laboratory, Batavia, Illinois 60510, USA \\ ${ }^{18}$ University of Florida, Gainesville, Florida 32611, USA \\ ${ }^{19}$ Laboratori Nazionali di Frascati, Istituto Nazionale di Fisica Nucleare, I-00044 Frascati, Italy \\ ${ }^{20}$ University of Geneva, CH-1211 Geneva 4, Switzerland \\ ${ }^{21}$ Glasgow University, Glasgow G12 8QQ, United Kingdom \\ ${ }^{22}$ Harvard University, Cambridge, Massachusetts 02138, USA \\ ${ }^{23}$ Division of High Energy Physics, Department of Physics, University of Helsinki and Helsinki Institute of Physics, \\ FIN-00014, Helsinki, Finland
}




\author{
${ }^{24}$ University of Illinois, Urbana, Illinois 61801, USA \\ ${ }^{25}$ The Johns Hopkins University, Baltimore, Maryland 21218, USA \\ ${ }^{26}$ Institut für Experimentelle Kernphysik, Universität Karlsruhe, 76128 Karlsruhe, Germany \\ ${ }^{27}$ Center for High Energy Physics: Kyungpook National University, Daegu 702-701, Korea; \\ Seoul National University, Seoul 151-742, Korea; \\ Sungkyunkwan University, Suwon 440-746, Korea; \\ Korea Institute of Science and Technology Information, Daejeon, 305-806, Korea; \\ Chonnam National University, Gwangju, 500-757, Korea \\ ${ }^{28}$ Ernest Orlando Lawrence Berkeley National Laboratory, Berkeley, California 94720, USA \\ ${ }^{29}$ University of Liverpool, Liverpool L69 7ZE, United Kingdom \\ ${ }^{30}$ University College London, London WC1E 6BT, United Kingdom \\ ${ }^{31}$ Centro de Investigaciones Energeticas Medioambientales y Tecnologicas, E-28040 Madrid, Spain \\ ${ }^{32}$ Massachusetts Institute of Technology, Cambridge, Massachusetts 02139, USA \\ ${ }^{33}$ Institute of Particle Physics: McGill University, Montréal, Canada H3A 2T8; \\ and University of Toronto, Toronto, Canada M5S $1 A 7$ \\ ${ }^{34}$ University of Michigan, Ann Arbor, Michigan 48109, USA \\ ${ }^{35}$ Michigan State University, East Lansing, Michigan 48824, USA \\ ${ }^{36}$ University of New Mexico, Albuquerque, New Mexico 87131, USA \\ ${ }^{37}$ Northwestern University, Evanston, Illinois 60208, USA \\ ${ }^{38}$ The Ohio State University, Columbus, Ohio 43210, USA \\ ${ }^{39}$ Okayama University, Okayama 700-8530, Japan \\ ${ }^{40}$ Osaka City University, Osaka 588, Japan \\ ${ }^{41}$ University of Oxford, Oxford OX1 3RH, United Kingdom \\ ${ }^{42}$ University of Padova, Istituto Nazionale di Fisica Nucleare, Sezione di Padova-Trento, I-35131 Padova, Italy \\ ${ }^{43}$ LPNHE, Universite Pierre et Marie Curie/IN2P3-CNRS, UMR7585, Paris, F-75252 France \\ ${ }^{44}$ University of Pennsylvania, Philadelphia, Pennsylvania 19104, USA \\ ${ }^{45}$ Istituto Nazionale di Fisica Nucleare Pisa, Universities of Pisa, Siena, and Scuola Normale Superiore, I-56127 Pisa, Italy \\ ${ }^{46}$ University of Pittsburgh, Pittsburgh, Pennsylvania 15260, USA \\ ${ }^{47}$ Purdue University, West Lafayette, Indiana 47907, USA \\ ${ }^{48}$ University of Rochester, Rochester, New York 14627, USA \\ ${ }^{49}$ The Rockefeller University, New York, New York 10021, USA \\ ${ }^{50}$ Istituto Nazionale di Fisica Nucleare, Sezione di Roma 1, University of Rome "La Sapienza," I-00185 Roma, Italy \\ ${ }^{51}$ Rutgers University, Piscataway, New Jersey 08855, USA \\ ${ }^{52}$ Texas A\&M University, College Station, Texas 77843, USA \\ ${ }^{53}$ Istituto Nazionale di Fisica Nucleare, University of Trieste/Udine, Italy \\ ${ }^{54}$ University of Tsukuba, Tsukuba, Ibaraki 305, Japan \\ ${ }^{55}$ Tufts University, Medford, Massachusetts 02155, USA \\ ${ }^{56}$ Waseda University, Tokyo 169, Japan \\ ${ }^{57}$ Wayne State University, Detroit, Michigan 48201, USA \\ ${ }^{58}$ University of Wisconsin, Madison, Wisconsin 53706, USA \\ ${ }^{59}$ Yale University, New Haven, Connecticut 06520, USA \\ (Received 23 October 2007; published 29 January 2008)
}

We report on the results of a search for standard model Higgs bosons produced in association with $W$ bosons from $p \bar{p}$ collisions at $\sqrt{s}=1.96 \mathrm{TeV}$. The search uses a data sample corresponding to approximately $1 \mathrm{fb}^{-1}$ of integrated luminosity. Events consistent with the $W \rightarrow \ell \nu$ and $H \rightarrow b \bar{b}$ signature are selected by triggering on a high- $p_{T}$ electron or muon candidate and tagging one or two of the jet candidates as having originated from $b$ quarks. A neural network filter rejects a fraction of tagged charm and light-flavor jets, increasing the $b$-jet purity in the sample. We observe no excess $\ell \nu b \bar{b}$ production beyond the background expectation, and we set $95 \%$ confidence level upper limits on the production cross section times branching fraction $\sigma(p \bar{p} \rightarrow W H) \operatorname{Br}(H \rightarrow b \bar{b})$ ranging from 3.9 to $1.3 \mathrm{pb}$, for specific Higgs boson mass hypotheses in the range 110 to $150 \mathrm{GeV} / c^{2}$, respectively.

DOI: 10.1103/PhysRevLett.100.041801

PACS numbers: $14.80 . \mathrm{Bn}, 13.85 . \mathrm{Rm}$

The standard model (SM) of elementary particle physics provides for electroweak gauge symmetry breaking via the Higgs mechanism [1], and the model predicts a single physical remnant of the added Higgs field. This remnant, the Higgs boson $H$, has yet to be observed experimentally.
Results from direct searches at the LEP collider exclude mass values less than $114.4 \mathrm{GeV} / c^{2}$ at a $95 \%$ confidence level [2], and global fits to precision electroweak data exclude masses greater than $144 \mathrm{GeV} / c^{2}$ at $95 \%$ confidence level [3]. For Higgs boson masses just above the 
range excluded by LEP, the decay to bottom quarks $b \bar{b}$ dominates. Even though gluon fusion $g g \rightarrow H \rightarrow b \bar{b}$ has the largest cross section among Higgs production processes in $p \bar{p}$ collisions [4], the $b \bar{b}$ data sample is dominated by a nonresonant multijet background. Consequently, we search for $W H$ production, requiring a leptonic $W$ boson decay to suppress the background. In this Letter we report results of a search for low-mass SM Higgs bosons produced in association with $W$ bosons and decaying to $b \bar{b}$ pairs.

Recent searches at CDF and D0 $[5,6]$ were limited not only by smaller data samples, but also by contamination from jets associated with charm or light quarks that are falsely tagged as $b$ jets. The search described in this Letter employs for the first time a neural network filter to reject such events, thereby improving the purity of the selected event sample. The data sample of $p \bar{p}$ collisions at $\sqrt{s}=$ $1.96 \mathrm{TeV}$ used here corresponds to $0.955 \pm 0.057 \mathrm{fb}^{-1}$ of integrated luminosity, nearly 3 times the sample used in previous searches.

The CDF II detector is a general-purpose detector located at the Tevatron $p \bar{p}$ collider at Fermilab. It consists of a cylindrical magnetic spectrometer surrounded by sampling calorimeters used to measure energies of electromagnetic showers and jets. Charged particle tracking is performed with microstrip silicon detectors surrounded by a large cylindrical multilayer drift chamber, both immersed in a solenoidal magnetic field. Jets are identified as a collection of hadronic and electromagnetic calorimeter towers, which are clustered using an iterative cone algorithm with a cone of $\Delta R=\sqrt{(\Delta \phi)^{2}+(\Delta \eta)^{2}}=0.4$ units in the azimuth-pseudorapidity space [7]. Planar drift chambers used for muon detection surround the calorimeters at least five interaction lengths from the interaction region.

Events compatible with the $\ell \nu b \bar{b}$ final state are selected by requiring exactly one electron or muon candidate and missing transverse energy $\mathbb{E}_{T}>20 \mathrm{GeV}$, after jets are corrected for detector imperfections and nonlinear calorimeter response [7]. The electron or muon must be within the central part of the detector, in the pseudorapidity regions $|\eta|<1.1$ or $|\eta|<1.0$, respectively, and must have transverse energy greater than $20 \mathrm{GeV}$. The lepton must be isolated from the rest of the event by a cone of radius $\Delta R=0.4$ containing no more than $10 \%$ of the lepton energy (excluding the lepton itself). To suppress background from $Z$ boson and diboson production, we reject events with more than one isolated lepton, as well as events in which the lepton and another high-energy track of opposite sign form an invariant mass between 76 and $106 \mathrm{GeV} / c^{2}$. Jets used in the analysis must fall within the acceptance of the silicon detector $(|\eta|<2.0)$ for reliable $b$ tagging, and they must have transverse energy greater than $15 \mathrm{GeV}$. Events with a $W$ and exactly 2 jets are used for this search, while other event samples with $W+1$ or $W+3,4$ jets are used for cross-checks of the background estimates.
A $B$ hadron, with relatively long lifetime and large mass, can decay to charged particles whose tracks have a large impact parameter, the distance of the closest approach to the interaction point in the transverse plane. Such tracks are fit to a secondary vertex, and the decay length of the $B$ hadron is defined as the distance between this vertex and the primary vertex. Specifically, we apply the SECVTX secondary vertex finding algorithm [8] to each jet in the event, using tracks within the $\Delta R=0.4$ cone centered on the jet axis. Three tracks with impact parameter significances greater than 2.0 are fit to a decay vertex. If this first pass fails, a second pass is attempted with two tracks having impact parameter significances greater than 3.0. Jets are $b$ tagged if the magnitude of the significance of the transverse decay length is greater than 7.5. Jets with a negative decay length have a reconstructed flight direction opposite the jet direction. This can happen when tracks coming from the primary vertex have significantly mismeasured impact parameters.

In addition to the secondary vertex finding algorithm, a neural network $(N N)$ filter has been trained with the JETNET program [9] to reject tagged jets originating from charm or light $(u, d, s)$ quarks. The $N N$ filter is composed of two networks in series, one to separate $b$ jets from light quark jets and the second to separate $b$ jets from $c$ jets. Both networks have the same set of 16 inputs: the number of tracks in the secondary vertex, the $\chi^{2}$ value of the vertex fit, the transverse decay length and its significance, the vertex mass calculated by assuming the charged pion mass for all particles, the proper time assuming the vertex mass, the fraction of the jet $p_{T}$ carried by tracks in the vertex, the vertex pass number, the number of tracks with significant impact parameter, the reconstructed mass of the SECVTX pass 1 and pass 2 tracks, the numbers of pass 1 and pass 2 tracks, the fraction of the jet $p_{T}$ carried by the pass 1 and pass 2 tracks, and finally the probability of a selected ensemble of tracks to have originated at the primary vertex [10]. The selection cuts on the $N N$ output are chosen to give $90 \%$ efficiency for true $b$ jets identified with the secondary vertexing algorithm. The response of the filter in simulated events has been verified using multijet data, and the rejection factors measured in simulated events are $65 \% \pm 5 \%$ for light-flavor jets and $50 \% \pm 5 \%$ for charm jets.

Our search criteria select events with exactly one highenergy charged lepton, missing transverse energy, and exactly two jets. The search sensitivity is maximized by defining two distinct subsamples based on the following $b$-tagging requirements: single-tagged events with exactly one $b$-tagged jet that passes the $N N$ filter, and doubletagged events with two $b$-tagged jets. The selected event sample includes contributions from other SM processes. The largest background rates are due to $W+$ jets production, $t \bar{t}$ production, and non- $W$ multijet production, with small contributions from electroweak boson production $W W$ or $W Z$. 
The dominant background contribution comes from $W+$ jets production, either with jets from $b$ or $c$ quarks or with jets mistagged by the $b$-tagging algorithm. The effect of true $W+$ heavy-flavor production is estimated from a combination of data and simulation. We use the ALPGEN Monte Carlo program [11] to calculate the rate of $W b \bar{b}, W c \bar{c}$, and $W c$ production relative to inclusive $W+$ jets production. Then this relative rate is applied to the observed $W+$ jets sample, after non- $W$ and $t \bar{t}$ contributions have been subtracted. Finally, we apply a $b$-tagging efficiency calculated using the appropriate ALPGEN event samples (corrected with the data-to-MC efficiency scale factor) and the $N N$ filter rejection rate.

Events from $t \bar{t}$ production followed by leptonic $W$ decay typically have two $b$ jets from $t$ decay, significant missing transverse energy, and one or two high-energy leptons with two or zero additional jets, depending on whether one or both $W$ bosons from the top quarks decay leptonically. The $t \bar{t}$ contribution to the $\ell \nu b \bar{b}$ final state is estimated using simulated PYTHIA events [12].

Multijet events may have high-energy identified leptons or missing transverse energies, both mimicking the signature of $W$ decay. These may be from semileptonic heavy flavor decay or from false reconstructions. The identified leptons from such events are rarely isolated in energy, as required by our event selection, and seldom yield large missing transverse energy. We therefore calculate the number of non- $W$ events in our selected sample by extrapolating from sideband regions (defined in the space of lepton energy isolation and missing transverse energy) into the signal region [13].

Contributions from events with falsely tagged lightflavor jets (mistags) are estimated by measuring a mistag rate in generic jet data. The mistag rate is further modified by the $N N$ filter efficiency. The resulting overall mistag rate is applied to the $W+$ jets sample to yield the number of mistagged events present in the sample.

The dominant uncertainty in the $W+$ heavy flavor background is the production rate calibration factor for simu- lation derived from multijet data [13]. Different simulation inputs give different factors, and we find a $35 \%$ relative error on the background from heavy flavor. The background from mistags has major uncertainties on the rate correction due to particle interactions in detector material and on the $N N$ rejection factor. Both are $15 \%$ relative errors.

We use the large $b$-tagged sample of $W+1$ jet events to derive a data-based scaling factor of $1.2 \pm 0.2$, which corrects a residual mismatch between the heavy flavor fraction correction factor in multijet data and the $W+$ jets sample. This single factor is applied to the $W+$ heavy flavor background calibration for all jet multiplicities, and it improves the agreement for the sideband multiplicities of $W+1,3,4$ jets. A summary of the estimated background contributions to the lepton + jets sample is shown in Table I, along with the results from the data sample.

Figures 1 and 2 show the dijet mass spectra in the singleand double-tagged 2-jet samples for the estimated background as well as for the observed events. A $115 \mathrm{GeV} / c^{2}$ Higgs boson signal at 10 times the SM rate is shown for comparison. There is no significant excess observed in the dijet mass spectrum. The largest discrepancy, for masses near $100 \mathrm{GeV} / c^{2}$, is less than 1 standard deviation defined by the uncertainty on the background estimate.

The acceptance for $W H \rightarrow \ell \nu b \bar{b}$, including leptonic $\tau$ decays, is calculated from samples generated with the PYTHIA Monte Carlo program using Higgs boson mass values between 110 and $150 \mathrm{GeV} / c^{2}$. The acceptances for the single $N N$ tag and double-tag selections are $1.3 \% \pm$ $0.1 \%$ and $0.4 \% \pm 0.1 \%$, including the $W$ branching ratio to lepton pairs, for a mass hypothesis of $115 \mathrm{GeV} / c^{2}$. The dominant systematic uncertainty on the acceptance is the $b$-tagging scale factor uncertainty, which is a $5.3 \%$ relative error for the single-tagged selection and a $16 \%$ relative error for the double-tagged selection. This value is largely due to uncertainties in fitting the $b / c$ ratio for the data sample in which the scale factor is measured. Additional

TABLE I. Background estimate for events with exactly one $N N b$ tag or double tag as a function of jet multiplicity.

\begin{tabular}{|c|c|c|c|c|c|c|c|}
\hline \multirow{2}{*}{$\begin{array}{c}\text { Selection } \\
\text { Jet Multiplicity }\end{array}$} & \multicolumn{4}{|c|}{ Single $N N$ tag } & \multicolumn{3}{|c|}{ Double tag } \\
\hline & 1 jet & 2 jets & 3 jets & $\geq 4$ jets & 2 jets & 3 jets & $\geq 4$ jets \\
\hline Mistag & $139.7 \pm 27.3$ & $53.9 \pm 10.7$ & $15.7 \pm 3.1$ & $4.2 \pm 0.8$ & $3.5 \pm 0.5$ & $2.0 \pm 0.3$ & $1.2 \pm 0.2$ \\
\hline$W b \bar{b}$ & $306.9 \pm 106.9$ & $144.7 \pm 49.4$ & $29.9 \pm 9.7$ & $6.4 \pm 2.5$ & $20.3 \pm 7.0$ & $5.7 \pm 1.8$ & $1.0 \pm 0.4$ \\
\hline$W c \bar{c}$ & $63.1 \pm 22.0$ & $43.0 \pm 14.7$ & $8.7 \pm 2.8$ & $1.9 \pm 0.8$ & $3.3 \pm 1.1$ & $0.4 \pm 0.1$ & $0.1 \pm 0.04$ \\
\hline$W c$ & $185.7 \pm 47.2$ & $34.4 \pm 9.0$ & $3.4 \pm 0.9$ & $0.6 \pm 0.2$ & $0.0 \pm 0.0$ & $0.0 \pm 0.0$ & $0.0 \pm 0.0$ \\
\hline$t \bar{t}(6.7 \mathrm{pb})$ & $6.9 \pm 1.2$ & $42.0 \pm 6.6$ & $84.9 \pm 12.8$ & $98.6 \pm 14.3$ & $10.4 \pm 2.3$ & $29.5 \pm 6.4$ & $45.5 \pm 9.9$ \\
\hline Single top & $16.7 \pm 1.8$ & $23.5 \pm 2.4$ & $4.8 \pm 0.5$ & $0.8 \pm 0.1$ & $4.2 \pm 0.7$ & $1.4 \pm 0.2$ & $0.3 \pm 0.1$ \\
\hline Diboson $/ Z^{0} \rightarrow \tau \tau$ & $11.7 \pm 2.2$ & $14.2 \pm 2.3$ & $3.9 \pm 0.9$ & $1.0 \pm 0.3$ & $1.2 \pm 0.3$ & $0.3 \pm 0.1$ & $0.1 \pm 0.1$ \\
\hline Non- $W$ QCD & $84.2 \pm 14.1$ & $38.9 \pm 6.7$ & $12.1 \pm 2.3$ & $5.5 \pm 1.2$ & $1.4 \pm 0.3$ & $0.9 \pm 0.2$ & $0.3 \pm 0.1$ \\
\hline Total background & $814.9 \pm 140.7$ & $394.4 \pm 66.6$ & $163.4 \pm 18.7$ & $118.9 \pm 14.9$ & $44.2 \pm 8.5$ & $40.1 \pm 6.8$ & $48.6 \pm 10.0$ \\
\hline Observed events & 856 & 421 & 177 & 139 & 39 & 44 & 65 \\
\hline
\end{tabular}




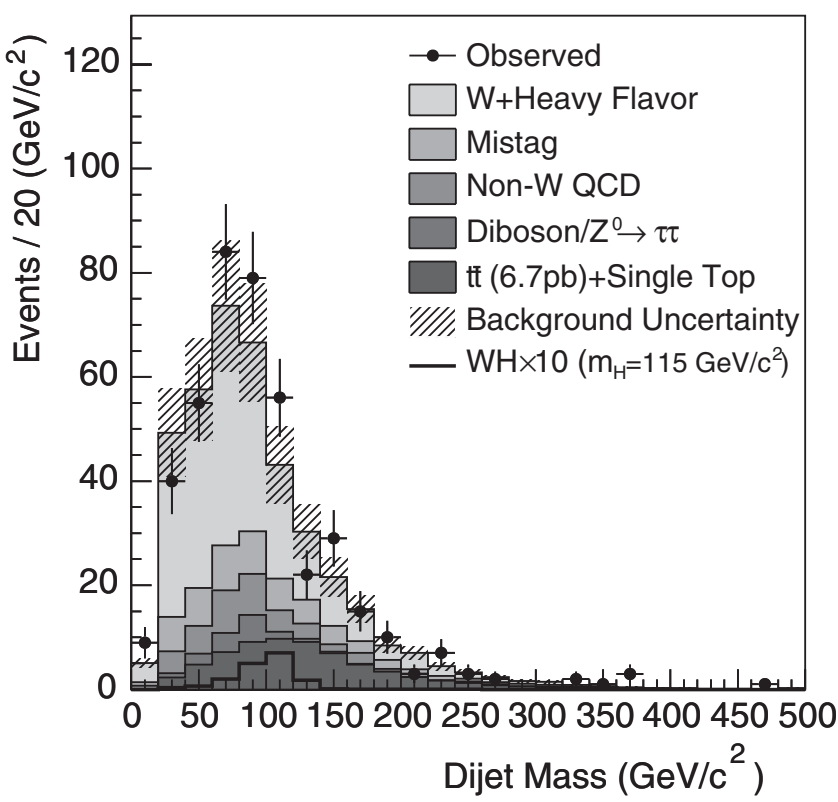

FIG. 1. Reconstructed dijet mass distributions for $W+2$-jet events with a single $b$ tag passing the $N N$ filter. The histogram binning is used in the binned likelihood calculation.

sources of systematic error include the jet energy scale, the lepton identification efficiency, and the initial and final state radiation models $[8,14]$.

Limits on the number of Higgs boson events, interpreted as the production rate times the branching fraction, are derived using a binned likelihood technique assuming

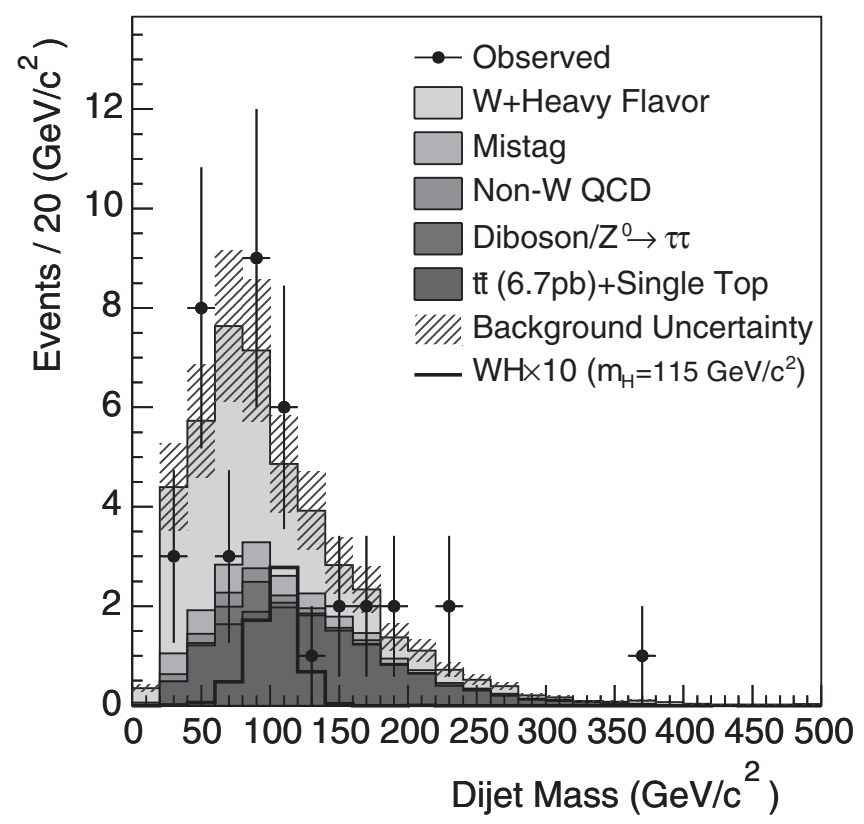

FIG. 2. Reconstructed dijet mass distributions for $W+2$-jet events with two $b$-tagged jets. The histogram binning is used in the binned likelihood calculation.
Poisson statistics. A Bayesian interval is constructed from the cumulative likelihood distributions and a prior probability density function uniform in the number of Higgs boson signal events $s$. The 95\% confidence level upper limit is defined to be the value $s_{\text {up }}$ for which $\int_{0}^{s_{\text {up }}} L(s) d s / \int_{0}^{\infty} L(s) d s=0.95$. The number of signal events is then converted to a Higgs boson production cross section times branching fraction $\sigma(p \bar{p} \rightarrow W H) \operatorname{Br}(H \rightarrow$ $b \bar{b})$.

The observed $95 \%$ confidence level upper limits on the cross section times branching fraction range from 3.9 to $1.3 \mathrm{pb}$, for Higgs boson mass hypotheses from 110 to $150 \mathrm{GeV} / c^{2}$, respectively. Figure 3 summarizes the observed limits as well as the expected limits as a function of the Higgs boson mass hypothesis. The observed limit in the low-mass region is roughly 2 standard deviations higher than the expected limit.

In this Higgs boson search, we have employed a novel neural network $b$-tagging filter on a data set nearly 3 times the size of previous searches. The resulting exclusion improves significantly the limits on the allowed production rate for Higgs bosons in $p \bar{p}$ collisions. Even though the largest improvement by far comes from the larger data set, separating the single- and double-tag samples results in a $20 \%$ improvement beyond the previous analysis, and rejecting charm and light-flavor jets with the $N N$ gains another $5 \%$ in sensitivity.

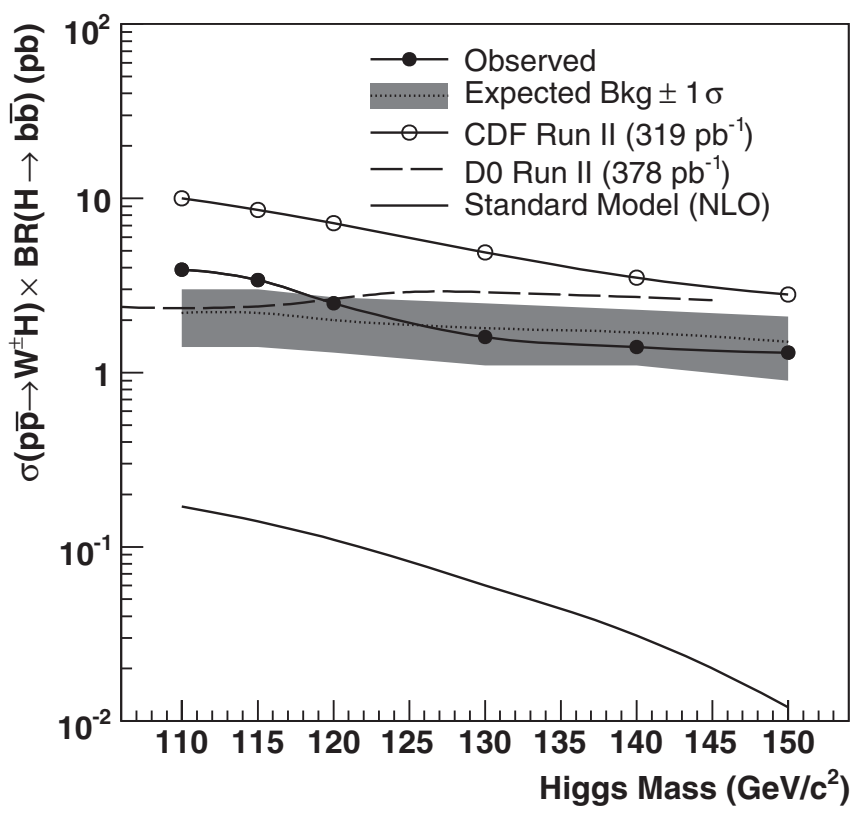

FIG. 3. The $95 \%$ confidence level upper limit on Higgs boson production cross section times branching fraction as a function of Higgs boson mass hypothesis. The expected limits from background-only pseudoexperiments are shown with the observed results from this and previous CDF and D0 searches. 
We thank the Fermilab staff and the technical staffs of the participating institutions for their vital contributions. This work was supported by the U.S. Department of Energy and National Science Foundation; the Italian Istituto Nazionale di Fisica Nucleare; the Ministry of Education, Culture, Sports, Science and Technology of Japan; the Natural Sciences and Engineering Research Council of Canada; the National Science Council of the Republic of China; the Swiss National Science Foundation; the A.P. Sloan Foundation; the Bundesministerium für Bildung und Forschung, Germany; the Korean Science and Engineering Foundation and the Korean Research Foundation; the Science and Technology Facilities Council and the Royal Society, U.K.; the Institut National de Physique Nucleaire et Physique des Particules/CNRS; the Russian Foundation for Basic Research; the Comisión Interministerial de Ciencia y Tecnología, Spain; the European Community's Human Potential Programme; the Slovak R\&D Agency; and the Academy of Finland.

${ }^{\mathrm{a}}$ With visitors from

${ }^{\mathrm{b}}$ University of Athens, 15784 Athens, Greece. ${ }^{\mathrm{c}}$ Chinese Academy of Sciences, Beijing 100864, China. ${ }^{\mathrm{d}}$ University of Bristol, Bristol BS8 1TL, United Kingdom. e University Libre de Bruxelles, B-1050 Brussels, Belgium. ${ }^{\mathrm{f}}$ University of California Irvine, Irvine, CA 92697, USA.

${ }^{\mathrm{g}}$ University of California Santa Cruz, Santa Cruz, CA 95064, USA.

${ }^{\mathrm{h}}$ Cornell University, Ithaca, NY 14853, USA.

${ }^{i}$ University of Cyprus, Nicosia CY-1678, Cyprus.

${ }^{j}$ University College Dublin, Dublin 4, Ireland.

${ }^{\mathrm{k}}$ University of Edinburgh, Edinburgh EH9 3JZ, United Kingdom.

${ }^{1}$ University of Heidelberg, D-69120 Heidelberg, Germany.

${ }^{\mathrm{m}}$ Universidad Iberoamericana, Mexico D.F., Mexico.

${ }^{\mathrm{n}}$ University of Manchester, Manchester M13 9PL, United Kingdom.

${ }^{\circ}$ Nagasaki Institute of Applied Science, Nagasaki, Japan.

${ }^{\mathrm{p}}$ University de Oviedo, E-33007 Oviedo, Spain.
${ }^{\mathrm{q}}$ Queen Mary, University of London, London E1 4NS, United Kingdom.

${ }^{\mathrm{r}}$ Texas Tech University, Lubbock, TX 79409, USA.

${ }^{\mathrm{s}}$ IFIC(CSIC-Universitat de Valencia), 46071 Valencia, Spain.

[1] P. W. Higgs, Phys. Rev. Lett. 13, 508 (1964).

[2] R. Barate et al. (ALEPH, DELPHI, L3, and OPAL Collaborations and the LEP Working Group for Higgs boson searches), Phys. Lett. B 565, 61 (2003).

[3] J. Alcaraz et al. (ALEPH, DELPHI, L3, and OPAL Collaborations and the LEP Electroweak Working Group), CERN Report No. CERN-PH-EP-2006-042, 2006.

[4] T. Han and S. Willenbrock, Phys. Lett. B 273, 167 (1991).

[5] A. Abulencia et al. (CDF Collaboration), Phys. Rev. Lett. 96, 081803 (2006).

[6] P. M. Jonsson (CDF and D0 Collaborations), in Proceedings of the 41st Rencontres de Moriond: $Q C D$ and Hadronic Interactions (The Gioi, Hanoi, 2006).

[7] The CDF reference frame uses cylindrical coordinates, where $z$ is the direction of the proton beam, and $\theta$ and $\phi$ are the polar and azimuthal angles with respect to the proton beam. The pseudorapidity is defined as $\eta=$ $-\ln \tan \theta / 2$, the transverse energy $E_{T}=E \sin \theta$, and the transverse momentum $p_{T}=p \sin \theta$. The $\mathscr{E}_{T}$ magnitude is the vector sum of all calorimeter deposits, projected into the transverse plane. The $\mathscr{E}_{T}$ vector is corrected for the energy deposition of the high-energy muons as well as for the jet energy corrections.

[8] A. Abulencia et al. (CDF Collaboration), Phys. Rev. Lett. 97, 082004 (2006).

[9] C. Peterson, T. Rognvaldsson, and L. Lönnblad, Comput. Phys. Commun. 81, 185 (1994).

[10] A. Abulencia et al. (CDF Collaboration), Phys. Rev. D 74, 072006 (2006).

[11] M. L. Mangano, M. Moretti, F. Piccinini, R. Pittau, and A. D. Polosa, J. High Energy Phys. 07 (2003) 001.

[12] T. Sjöstrand et al., Comput. Phys. Commun. 135, 238 (2001).

[13] D. Acosta et al. (CDF Collaboration), Phys. Rev. D 71, 052003 (2005).

[14] A. Bhatti et al. (CDF Collaboration), Nucl. Instrum. Methods Phys. Res., Sect. A 566, 375 (2006). 